\title{
Left Ventricular Mass Index as an Outcome Measure in Clinical Trials in Dialysis Patients: A Word of Caution
}

\author{
Carmine Zoccali \\ Nephrology, Dialysis and Transplantation Unit, and CNR-IBIM, Clinical Epidemiology and Pathophysiology of \\ Renal Diseases and Hypertension, Reggio Calabria, Italy
}

Left ventricular hypertrophy $(\mathrm{LVH})$ represents one of the strongest risk factors of death and major cardiovascular (CV) events both in the general population and in patients with cardiac diseases. This biomarker is a powerful integrator of the long-term exposure of the myocardium to environmental risk factors like pressure and volume/salt overload, metabolic derangements including insulin resistance and hyperinsulinemia, sympathetic overactivity and genetic factors [1]. Importantly, the presence of LVH predicts not only the risk for cardiac events like myocardial infarction or heart failure, but also the risk of cerebrovascular events. LVH is endowed with an important characteristic required by prognostic biomarkers, that is, sensitivity to therapeutic interventions which reduce CV risk [2]. It is indeed well demonstrated that regression of LVH brought about by optimal blood pressure control, weight loss and low salt intake is accompanied by a parallel decrease in the risk for $\mathrm{CV}$ complications, thus making $\mathrm{LVH}$ a valid instrument for risk monitoring.

Increases in LV mass are associated with higher CV risk. They are commonly associated with pathological conditions such as obesity and hypertension, and repre- sent an adaptation of the heart to accommodate an increased burden of pumping $[3,4]$.

$\mathrm{LVH}$ is common in patients with end-stage renal disease (ESRD) (prevalence 70-80\%) and entails an ominous prognosis $[5,6]$. Partial regression of LVH may occur after long-term control of arterial hypertension and/ or very severe anemia and after kidney transplantation. Because it is sensitive to ongoing clinical interventions and for its strong prognostic power, measurement of LV mass is now increasingly adopted as a main outcome measure in intervention studies in ESRD [7, 8]. The two geometric patterns (concentric and eccentric) of LVH are almost equally frequent in this population [6] and concentric and eccentric LVH carry a similar risk excess for death in this population.

In this issue, Rajiv Agarwal and colleagues [9] report on the short-term evolution of LVH in patients who took part in the Dry-Weight Reduction in Dialysis Patients (DRIP) trial [10], a remarkable study documenting that ultrafiltration (UF) intensification allows better control of hypertension in this high-risk population. LV mass did not change over time in control patients but showed a clear-cut decrease $\left(7.4 \mathrm{~g} / \mathrm{m}^{2}, 5.4 \%\right)$ in those who un-

\section{KARGER}

Fax +41613061234 E-Mail karger@karger.ch www.karger.com
(C) 2011 S. Karger AG, Basel

0250-8095/11/0334-0370\$38.00/0

Accessible online at:

www.karger.com/ajn
Carmine Zoccali

Nefrologia e CNR, Ospedali Riuniti

IT-89124 Reggio Calabria (Italy)

Tel. +390965 397010

E-Mail carmine.zoccali@tin.it 
Fig. 1. Effect of volume removal during dialysis on the estimate of the left ventricular mass index (LVMI) by the Devereux formula. A $0.4-\mathrm{cm}$ decrease in the left ventricular end-diastolic diameter (LVEDD: from 5.8 to $5.4 \mathrm{~cm}$ ) is the sort of reduction in LVEDD commonly observed in a patient with a normal BSA $\left(1.73 \mathrm{~m}^{2}\right)$ after a volume removal of about 3 liters. IVST = Interventricular septum thickness $(\mathrm{cm})$; $\mathrm{PWT}=$ posterior wall thickness $(\mathrm{cm})$.

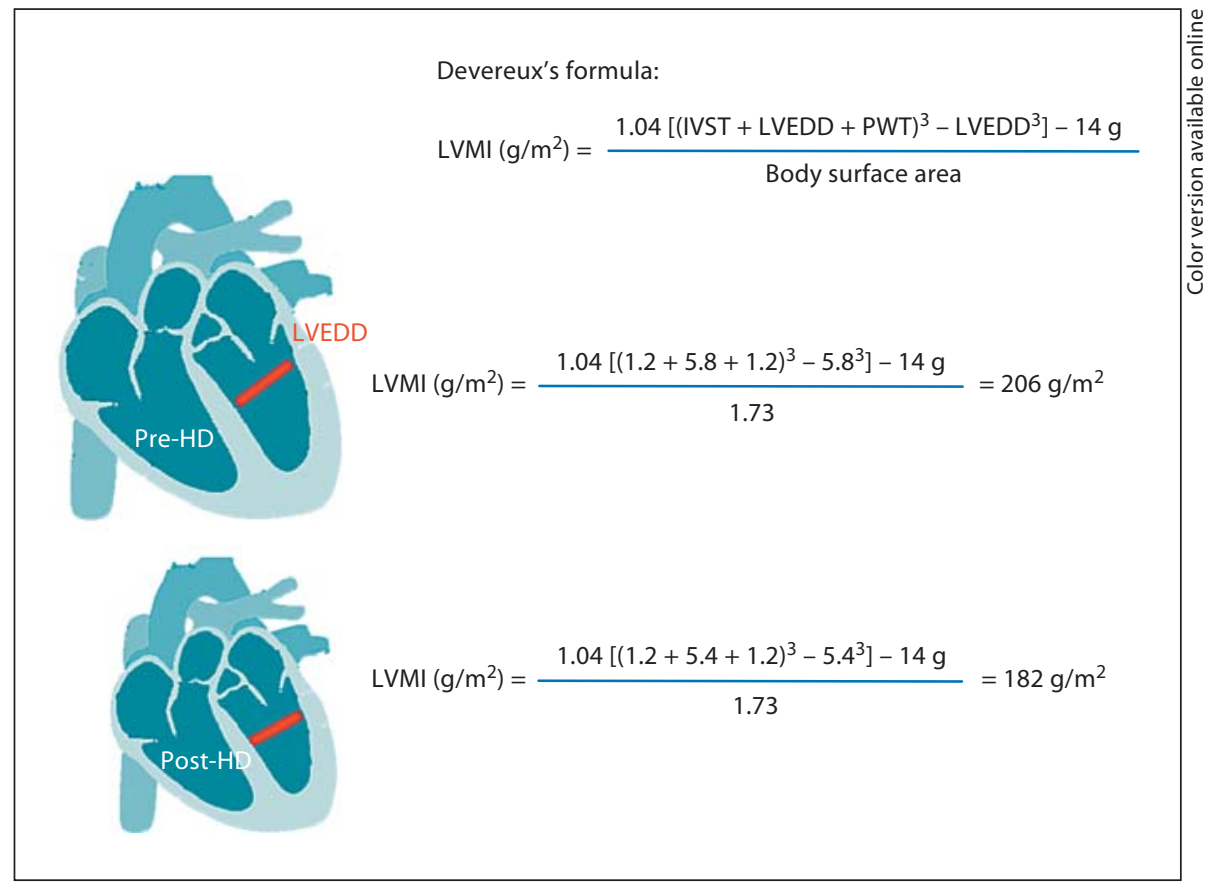

derwent UF intensification. Furthermore, patients who started the study with a LV mass greater than the median value in the same population manifested a substantially greater reduction in interdialysis ambulatory $\mathrm{BP}(-16 \mathrm{~mm}$ $\mathrm{Hg}$ ) than those with baseline LV mass below the median $(-2.5 \mathrm{~mm} \mathrm{Hg})$, suggesting that the degree of volume expansion predicts the magnitude of the $\mathrm{BP}$ response to UF in dialysis patients.

Given the high predictive power of LV mass for death and $\mathrm{CV}$ events, prima facie, the parallel decline in BP and in LV mass may suggest that UF intensification - a purely technical, easily implementable intervention - may translate into better clinical outcomes in ESRD patients. If this is the case, UF intensification could represent a clinically important, cheaper and much less demanding alternative to frequent dialysis to improve the ominous prognosis of these patients. Indeed, LVH regression in DRIP was of the same order as that registered in the Frequent Hemodialysis study [8], a long-term clinical trial where volume removal was diluted over a much longer dialysis time. However, interpreting the decrease in LV mass observed in DRIP as a favorable prognostic sign is problematic both because the time frame of the study was too short (just 2 months) and because the peculiar type of geometric rearrangement of the LV underlining the decrease in LV mass observed in this study. Actually, the reduction in LV mass mainly depended on a decrease in the LV end-diastolic diameter (cavitary component). Volume removal has a relevant influence on the measurement's LV mass which shows a substantial fall across a single dialysis session, an effect entirely attributable to the decrease of the end-diastolic diameter (fig. 1). Predictably, UF intensification in the DRIP study reduced blood volume and the cavitary component of LV mass but failed to modify the muscular component. LV mass as an outcome measure in clinical trials in hemodialysis patients should be interpreted with caution. In context of the present trial, short-term improvements in BP and in LVH achieved by UF intensification should not necessarily be seen as a guarantee of better clinical outcomes. In a recent secondary analysis of the Hemodialysis Study [11], patients with the highest UF rates had higher allcause and CV mortality, particularly patients with heart failure, i.e. a substantial proportion (about 20\%) of today's dialysis population.

Achievement and maintenance of dry-weight by UF and adherence to low salt diet induced a remarkable decline $(-23 \%)$ in the muscular component (posterior wall thickness) of the LV along with a reduction in LV enddiastolic diameter in a small, uncontrolled study with a 1.5-year follow-up [12]. The Frequent Hemodialysis trial [8] and the Canadian study of nocturnal dialysis [7] have convincingly demonstrated that augmenting dialysis duration, an intervention which dilutes volume removal 
over a longer time, has a favorable effect on survival and on LV mass. As emphasized elsewhere by Agarwal et al. [10], whether pure UF intensification at a constant dialysis time may allow better clinical outcomes still remains an open question with major clinical and financial implications. Thus, the intriguing observations in this second- ary analysis of DRIP, rather than proving the effectiveness of UF intensification for reducing the high risk of hypertension in ESRD, provide an additional argument for prioritizing clinical trials based on clinical end-points (mortality, CV events, AV fistula complications) to definitively test the clinical value of this intervention.

\section{References}

1 Devereux RB, de Simone G, Ganau A, Roman MJ: Left ventricular hypertrophy and geometric remodeling in hypertension: stimuli, functional consequences and prognostic implications. J Hypertens Suppl 1994; 12:S117-S127.

2 Muiesan ML, Salvetti M, Rizzoni D, Monteduro C, Castellano M, Agabiti-Rosei E: Persistence of left ventricular hypertrophy is a stronger indicator of cardiovascular events than baseline left ventricular mass or systolic performance: 10 years of follow-up. J Hypertens Suppl 1996;14:S43-S49.

3 Drazner MH: The progression of hypertensive heart disease. Circulation 2011;123:327334.

4 Muiesan ML, Salvetti M, Monteduro C, Bonzi B, Paini A, Viola S, Poisa P, Rizzoni D, Castellano M, Agabiti-Rosei E: Left ventricular concentric geometry during treatment adversely affects cardiovascular prognosis in hypertensive patients. Hypertension 2004; 43:731-738
5 Foley RN, Parfrey PS: Cardiac disease in chronic uremia: clinical outcome and risk factors. Adv Ren Replace Ther 1997;4:234248.

6 Zoccali C, Benedetto FA, Mallamaci F, Tripepi G, Giacone G, Cataliotti A, Seminara G, Stancanelli B, Malatino LS: Prognostic impact of the indexation of left ventricular mass in patients undergoing dialysis. J Am Soc Nephrol 2001;12:2768-2774.

7 Culleton BF, Walsh M, Klarenbach SW, Mortis G, Scott-Douglas N, Quinn RR, Tonelli M, Donnelly S, Friedrich MG, Kumar A, Mahallati H, Hemmelgarn BR, Manns BJ: Effect of frequent nocturnal hemodialysis vs. conventional hemodialysis on left ventricular mass and quality of life: a randomized controlled trial. JAMA 2007;298:1291-1299.

8 Chertow GM, Levin NW, Beck GJ, Depner TA, Eggers PW, Gassman JJ, Gorodetskaya I, Greene T, James S, Larive B, Lindsay RM, Mehta RL, Miller B, Ornt DB, Rajagopalan S, Rastogi A, Rocco MV, Schiller B, Sergeyeva O, Schulman G, Ting GO, Unruh ML, Star RA, Kliger AS: In-center hemodialysis six times per week versus three times per week. N Engl J Med 2010;363:2287-2300.
9 Agarwal R, Bouldin J, Light R, Garg A: Probing dry-weight improves left ventricular mass index; Am J Nephrol 2011;33:373-380.

10 Agarwal R, Alborzi P, Satyan S, Light RP: Dry-weight Reduction In hypertensive hemodialysis Patients (DRIP): a randomized, controlled trial. Hypertension 2009;53:500507.

11 Flythe JE, Kimmel SE, Brunelli SM: Rapid fluid removal during dialysis is associated with cardiovascular morbidity and mortality. Kidney Int 2011;79:250-257.

12 Ozkahya M, Toz H, Qzerkan F, Duman S, Ok E, Basci A, Mees EJ: Impact of volume control on left ventricular hypertrophy in dialysis patients. J Nephrol 2002;15:655-660. 